\title{
Bioanalysis
}

\section{2nd International Symposium on Microscale Separations and Bioanalysis (MSB 2016)}

\section{2nd International Symposium on Microscale Separations and Bioanalysis, Niagara-on-the-Lake, Canada, 3-7 April 2016}

The 32nd edition of the International Symposium on Microscale Separations and Bioanalysis (MSB) was held in Niagara-on-the-Lake, Canada, from 3 to 7 April 2016. This article outlines the aim and the main distinctive features of the MSB symposium. A selection of the scientific research presented at MSB 2016 is highlighted with a special focus on microscale separation developments in the fields of sample preparation, (glyco)proteomics and metabolomics.

First draft submitted: 31 May 2016; Accepted for publication: 22 June 2016;

Published online: 15 July 2016

Keywords: award/ MSB 2017 • Beckman medal • international symposium • MSB 2016

- scientific highlights

\section{Background}

The 32nd International Symposium on Microscale Separations and Bioanalysis (MSB), which was held in Niagara-on-theLake, Canada, from 3 to 7 April 2016, was chaired by $\mathrm{P}$ Britz-McKibbin (McMaster University, Hamilton, Canada) with S Krylov (York University, Toronto, Canada) and K Waldron (Université de Montréal, Montreal, Canada) as conference cochairs (further details can be found at the MSB 2016 symposium website [1]). Originally starting as the International Symposium on High Performance Capillary Electrophoresis in Boston in 1989, the meeting evolved as the MicroScale Bioseparations (MSB) series in order to include developments of related techniques, such as, for example, miniaturized LC, microfluidic separations and lab-on-achip technology. To broaden the scope of the MSB series to a wider range of scientists, and to better represent the application areas in which microscale separation techniques and methods are developed and used, the stra- tegic program committee of MSB changed MicroScale Bioseparations into Microscale Separations and Bioanalysis, which was used as the official conference name for MSB 2016 in Niagara-on-the-Lake.

The discussion of cutting-edge research on the frontiers of microscale separation science could be considered a core theme of MSB 2016. The meeting especially stimulated the exchange of ideas of unpublished scientific work in a confidential setting, thereby following a Gordon Research Conference format. Roughly $70 \%$ of the scientific program has been constructed on the basis of contributed abstracts, where a double-blind peerreview assessment was followed to ensure that high-quality scientific work was presented. A strong feature of MSB is the sufficient time scheduled for the scientific discussion of each lecture.

\section{An overview of selected highlights}

Roughly 230 participants from academia, pharmaceutical and biotechnology indus-
Rawi Ramautar

Division of Analytical Biosciences, Leiden Academic Center for Drug Research, Leiden University, Einsteinweg 55, 2333 CC Leiden, The Netherlands r.ramautar@lacdr.leidenuniv.nl 
tries, government and analytical companies joined the MSB 2016 symposium to discuss up-to-date developments in the field of microscale separation techniques and methods. The meeting started with presymposium short courses covering topics like sample pretreatment, advances in capillary electrophoresis-MS for characterization of biologics and multivariate data analysis for metabolomics, suited for both beginners and advanced separation scientists. The scientific lectures were scheduled over 5 days, starting with the opening ceremony in which the conference chair emphasized the value of an active scientific discussion, and specific time was reserved for this purpose after each lecture. The scientific program started by two plenary lectures in which the important role of the development of separation techniques for untargeted analysis of small molecules and adducts in biospecimens from diseased and healthy subjects with the goal of discovering disease causes was highlighted. The talks were followed by an open and active discussion, for example, the strengths and limitations of hydrophilic interaction LC (HILIC) for metabolic profiling were debated. The following 4 days comprised a diverse spread of scientific sessions ranging from advances in sample preparation, hyphenated techniques, microfluidic designs, ion mobility MS and affinity separations to application areas such as (bio) pharmaceutical analysis, chiral separations, (glyco) proteomics, metabolomics and environmental analysis arranged in two parallel tracks with keynote and contributed lectures.

\section{Future stars in microseparations}

A special feature of MSB 2016 was the session on Future Stars in Microseparations in which research contributions from young investigators/early career researchers with a focus on the basic development and/or innovative applications of microscale separations for bioanalysis were highlighted. For example, D Cabooter (University of Leuven, Leuven, Belgium) proposed a new procedure to study mass transfer phenomena in HILIC and reversed-phase LC (RPLC) under identical packing conditions. This procedure required a thorough characterization of column performance and packing characteristics under RPLC conditions, followed by removal of the stationary phase to obtain a bare silica column which could be evaluated under HILIC conditions. The study revealed that band broadening originating from eddy dispersion is significantly higher under HILIC conditions as compared with RPLC, thereby indicating that the observed eddy dispersion was considerably affected by the interior structure and the retention mechanism of the particles.

\section{Sample preparation}

The development of selective preconcentration techniques with a possibility for automation was a major theme during the sample preparation session. J Schappler (University of Geneva, Geneva, Switzerland) presented a novel dynamic-electromembrane extraction device for the extraction of neuropeptides from cerebrospinal fluid. D Fuchs (University of Copenhagen, Copenhagen, Denmark) reported the implementation of electromembrane extraction (EME) into an autosampler for high-throughput analysis of samples by EME-LC-MS. It was shown that by flushing the EMEsyringe with acidic wash buffer and reverting the applied electric potential, carryover between samples could be reduced to below $1 \%$. By making use of the high-extraction speed of EME, a complete analytical workflow of purification, separation and analysis of sample could be achieved within $6 \mathrm{~min}$.

\section{Biologics \& glycan analysis}

In the biologics and glycan analysis session, G Kammeijer (Leiden University Medical Center, Leiden, The Netherlands) demonstrated the impact of using a dopant enriched nitrogen gas in sheathless CE-MS for glycopeptide analysis. Using acetonitrile as a dopant, an increased sensitivity was observed as compared with conventional sheathless CE-MS. Up to 25-fold higher sensitivities for model glycopeptides were obtained, allowing for limits of detection which have not been obtained by stateof-the-art nano-LC-MS [2]. Apart from the sensitivity enhancement the use of dopant enriched nitrogen gas also improved the repeatability of the method. C Neusüß (Aalen University, Aalen, Germany) gave an overview of the possibilities of CE-MS and online 2D CE coupled to MS for glycan and intact protein analysis.

\section{Metabolomics \& proteomics}

O Fiehn (University of California, CA, USA) highlighted the complexity of the metabolome and the need for reliable, high-efficiency separation techniques for global metabolic profiling, in his plenary lecture about second-generation metabolomics. Apart from the metabolites originating from classical endogenous biochemical pathways, attention was also devoted to metabolites originating from microbes, food, xenobotics and the epimetabolome, which deals with modified metabolites with specific biological functions. Emphasis was also given to the critical use of (freely available) data preprocessing and analysis software for the evaluation of recorded metabolomics data. A DiBattista (McMaster University, Hamilton, Canada) presented a novel high-throughput CE-MS platform for the screening of novel metabolic signatures in affected cystic fibrosis (CF) newborns relative to healthy controls 
in order to improve overall screening performance, as the current approach for the screening of CF is limited by a high rate of false-positives. Global metabolic profiling of dried blood spots by CE-MS revealed significant metabolic differences in affected CF patients as compared with healthy subjects. Therefore, this work may provide insight into the molecular mechanisms of disease pathogenesis in CF and new targets for therapeutic intervention. A Ivanov (Norteastern University, MA, USA) proposed an integrated platform of sample procurement, preparation and analysis for indepth proteomics of rare cells in whole blood. For this, microfluidic magnetophoretic isolation of target cells spiked into blood at the level of 1000-2000 cells/ml, followed by focused acoustics-based sample preparation has been coupled with a porous layer open tubular RPLC-MS method [3]. The resulting zeptomole detection sensitivity allowed the identification of roughly 4000 proteins using an injection volume which equals 100-200 cells per analysis. The development of novel interfacing techniques for hyphenating CE to MS was a major theme during the CE-MS session, emphasizing the need of using the intrinsically low-flow separation property of $\mathrm{CE}$, under which conditions highresolution electrophoretic separations can be obtained, in an effective way with ESI-MS.

\section{Arnold O Beckman medal \& award}

An important highlight in the scientific program was the Arnold O Beckman Medal and Award for outstanding scientific achievements in the field of electrodriven separation techniques, which was presented this year to Bohuslav Gaš (Charles University, Prague, Czech Republic) by J Chapman (Sciex) for his contribution to mathematical modeling of electromigration processes. Software tools based on this modeling work like SIMUL and Peak Master have played a key role $\mathrm{CE}$ method development and problem solving.

\section{Posters \& 3-min talks}

Though poster sessions could be considered a key part of a scientific meeting, the number of poster presenta-

\section{References}

1 Microscale Separations and Bioanalysis (MSB) 2016 symposium. www.msb2016.org

2 Kammeijer GS, Kohler I, Jansen BC et al. Dopant enriched nitrogen gas combined with sheathless capillary electrophoresis-electrospray ionization-mass spectrometry for improved sensitivity and repeatability in glycopeptide analysis. Anal. Chem. 88(11), 5849-5856 (2016). tions was rather limited at MSB 2016. A nice feature was the 3-min talk sessions during which young scientists could showcase their research project. There were significantly more scientific lectures as poster presentations. During breaks and social events there was ample time for further discussions, exchange of ideas and networking. The open atmosphere of the meeting and the willingness to share information among all participants were part of the success of MSB 2016.

\section{MSB 2017}

In 2017, the 33rd edition of MSB will be organized in Noordwijkerhout, The Netherlands 26-29 March, by G Somsen (VU University, Amsterdam, The Netherlands) and R Ramautar (Leiden University, Leiden, The Netherlands) as conference chairs. Further details can be found at the MSB 2017 symposium website [4]. Overall, MSB is a meeting where advancements in microscale separation techniques and approaches in a broad range of application fields are presented and discussed in an open and stimulating ambience, ensuring vigorous scientific exchange among delegates and it allows to initiate research collaborations during excellent social program activities.

\section{Disclaimer}

R Ramautar was a session chair responsible for the proteomics, metabolomics and capillary electrophoresis-mass spectrometry sessions at MSB 2016 and will be co-organizing the 2017 meeting.

\section{Financial \& competing interests disclosure}

The author has no relevant affiliations or financial involvement with any organization or entity with a financial interest in or financial conflict with the subject matter or materials discussed in the manuscript. This includes employment, consultancies, honoraria, stock ownership or options, expert testimony, grants or patents received or pending, or royalties.

No writing assistance was utilized in the production of this manuscript.

3 Li S, Plouffe BD, Belov AM et al. An integrated platform for isolation, processing, and mass spectrometry-based proteomic profiling of rare cells in whole blood. Mol. Cell Proteomics 14(6), 1672-1683 (2015).

4 Microscale Separations and Bioanalysis (MSB) 2017 symposium. www.msb2017.org 